\author{
Dechen W. Tshering Vogel \\ Lynne S. Steinbach \\ Ralph Hertel \\ Juerg Bernhard \\ Edouard Stauffer \\ Suzanne E. Anderson
}

\section{Acromioclavicular joint cyst: nine cases of a pseudotumor of the shoulder}

Received: 13 August 2004

Revised: 17 October 2004

Accepted: 27 October 2004

Published online: 19 February 2005

(C) ISS 2005

D. W. Tshering Vogel ( $)$ S. E. Anderson Department of Diagnostic, Interventional and Pediatric Radiology,

University Hospital of Bern,

3010 Bern, Switzerland

e-mail: dechen.tshering@insel.ch

Tel.: + 41-031-6322527

Fax: +41-031-6324874

\section{S. Steinbach}

Department of Radiology,

University of California San Francisco,

San Francisco, California, USA

R. Hertel

Department of Orthopedics,

Plastic and Hand Surgery,

University Hospital of Bern,

Bern, Switzerland

J. Bernhard

Department of Rheumatology,

Burgerspital,

Solothurn, Switzerland

E. Stauffer

Department of Pathology,

University Hospital of Bern,

Bern, Switzerland
Abstract Objective: (1) To analyse the imaging appearances of nine patients with acromioclavicular joint cysts presenting as shoulder masses for tumor staging with operative, histopathological and joint aspiration findings. Design and patients: Retrospective review of imaging and correlation with clinical, operative and surgical notes. Images were reviewed by two musculoskeletal radiologists by consensus. Nine patients who presented clinically with a shoulder mass were evaluated by radiographs $(n=9)$, ultrasound $(n=1)$, conventional arthrography $(n=3)$, MRI ( $n=6$; with direct MR arthrography $n=2$, indirect MR arthrography $n=4)$. Results: All patients had a focal mass superior to the AC joint, with a size ranging from $1.5 \mathrm{~cm}$ to $6 \mathrm{~cm}$ and a mean of $3.27 \mathrm{~cm}$. Correlation was available with surgery $(n=7)$, histopathology $(n=2)$ and cyst aspiration $(n=2)$. Two patients were managed conservatively. Geyser sign was positive in all three arthrograms. All MRIs revealed extensive rotator cuff tears with a column of fluid extend- ing from the glenohumeral joint through the rotator cuff tear into the acromioclavicular joint and acromioclavicular cyst. Chondrocalcinosis was seen in the acromioclavicular joint cyst $(n=2)$ and in the glenohumeral joint $(n=1)$. Aspirate in two patients contained calcium pyrophosphate dihydrate crystals. Conclusion: Acromioclavicular joint cysts may present as a tumor mass. They are associated with extensive rotator cuff tears and there is usually communication of the cyst with the joint space. This feature excludes a diagnosis of tumor. AC joint cysts may be associated with calcium pyrophosphate dihydrate deposition disease.

\section{Keywords Conventional} arthrography · MRI · Geyser sign · Rotator cuff tear - Acromioclavicular joint cyst $\cdot$ Pseudotumour

\section{Introduction}

Acromioclavicular joint cysts (AC joint cysts) have been reported in small series in the orthopaedic literature in association with extensive rotator cuff tears $[1,2,3,4]$. The earliest cases of AC cysts in association with rotator cuff tears were described by Craig in 1984 and 1986 [1, 2]. Marino et al. in1998 [3] found only 16 cases of subcutaneous cysts located over the $\mathrm{AC}$ joint region in the literature. Thirteen of 16 were associated with extensive rotator cuff tears [3]. The importance of this association lies in the high recurrence rate after simple excision of the cyst if the tear and joint pathology are not simultaneously treated [3]. We present nine patients with an initial clinical diagnosis of a soft tissue tumor of the shoulder region. Upon imaging, these masses were caused by an AC joint cyst related to the extensive rotator cuff tear. 


\section{Materials and methods}

Patients

Patient's ages ranged from 57 to 86 years with an average age of 67.7 years. There were two females and seven males. The referral basis was rheumatology and orthopaedic clinics. All patients presented with shoulder region masses for tumor imaging. Six of the nine patients were referred to the ambulatory MRI unit for imaging by the final author between the years 1996 and 2003. Three patients were referred for a second opinion. Between 1996 and 2003, 77 patients were referred for MRI of the shoulder for tumor imaging. There were no other lesions centered over the AC joint but one patient had subcutaneous fat necrosis adjacent to the AC joint due to chronic occupational injury. All our patients had clinically evident soft tissue masses over the AC joint (Figs. 1A, 3A) with a size ranging from $1.5 \mathrm{~cm}$ to $6 \mathrm{~cm}$. Seven patients had pain. On average, the duration of symptoms and swellings was two years, however in one of the patients with calcium pyrophosphate dihydrate (CPPD) crystal deposition disease, symptoms were more acute, increasing over two weeks. On clinical examination, the swelling was firm and fixed to the underlying tissues with freely mobile overlying skin in all cases. Two patients had a limited range of movement of the glenohumeral joint.

\section{Imaging}

Twenty-seven radiographs were available for review. All patients underwent three-plane conventional radiography (anteroposterior, axial and Neer views). The latter was performed to project the humerus and scapula over each other and free from the chest wall with a $20^{\circ}$ rotation of the patient in the PA position. The images were reviewed by two musculoskeletal radiologists by consensus. Radiographic features analysed were diameter and location of the soft tissue mass, presence of calcifications, acromion type according to Bigliani et al. [5], and changes in the acromioclavicular and glenohumeral joints. An AC joint space between 1 and $3 \mathrm{~mm}$ was taken as normal with an extreme range of 0.5 to $7 \mathrm{~mm}$ [6]. The degenerative changes in the glenohumeral joint were classified subjectively as mild, moderate and severe. The presence of small osteophytes with a normal joint space was classified as mild, the presence of larger osteophytes and slight joint space narrowing as moderate, and the presence of marked osteophytes with decreased joint space was classified as severe. The subacromial space was reviewed, with a decreased subacromial space defined as less than $7 \mathrm{~mm}$ acromiohumeral distance [7].

MR features reviewed included the status of the AC joint and the rotator cuff. The site and extent of rotator cuff tear were analysed and grading was performed for fatty muscle atrophy [8]. The integrity of the superior and inferior AC joint capsule was assessed with integrity being defined as a continuity of these structures and rupture as a lack of continuity with fluid passing between the free edges. Specifically, on MRI we looked for a column of fluid extending from the glenohumeral joint into the $\mathrm{AC}$ joint and $\mathrm{AC}$ joint cyst.

Four patients underwent MR imaging on a $1.5 \mathrm{~T}$ machine (General Electric, Milwaukee). A dedicated receive only shoulder coil was used. Standard protocols were followed. Imaging sequences included axial T1 weighted FSE (TR 400-600, TE 15-20), oblique coronal proton density and T2 weighted FSE (TR 34006000, PD: TE 20-30, T2: TE 85-120), sagittal Short Tau Inversion Recovery (STIR) (TR 3400-6000, TE 15-20, TI: 150m) and T1 weighted fat suppressed imaging after intravenous gadolinium injection, in all three planes (TR 400-600, TE 15-30) as part of an indirect MR arthrography technique. A $4 \mathrm{~mm}$ slice thickness with $1 \mathrm{~mm}$ slice gap was used, with a matrix of $512 \times 256$, and the number of excitations was 2-4. Two patients underwent direct MR arthrography with standard protocol of 3-plane T1 weighting with fat suppression and oblique coronal $\mathrm{T} 2$ weighting.

One patient underwent ultrasonography and conventional arthrography. Seven patients underwent surgery, with operative correlation in all seven, and two went on to have histological correlation. Aspiration of the swelling was performed in two patients, and these two patients subsequently underwent surgery due to recurrence. Surgical and radiological correlation was made by discussion with the surgeons and with surgical reports. At the patients request, two patients were managed conservatively and no surgical correlation is available in these two patients; however, the imaging features had excluded a tumor and at clinical follow up the cosmetic deformity and shoulder dysfunction no longer concerned the patients.

\section{Results}

On radiographs, all patients had a soft tissue swelling over the AC joint (Fig. 2A) with a size between $1.5 \mathrm{~cm}$ to $6 \mathrm{~cm}$ and a mean size of $3.27 \mathrm{~cm}$ in maximal diameter. The acromioclavicular joint space measured between 2 and $6 \mathrm{~mm}$, which was within the normal range [6]. All AC joint borders were smooth. Inferior acromial osteophytes were present in five patients and measured between 1 and $2 \mathrm{~mm}$. Inferior clavicular osteophytes were present in seven patients and measured 1 to $2 \mathrm{~mm}$. Superior clavicular osteophytes were present in three patients and measured between 1 and $2 \mathrm{~mm}$. Due to the small size of the osteophytes and joint spaces in the normal range, the AC joint changes were classified as mild. All patients had a type II acromion except one who had a type III acromion [5]. One patient had an os acromiale. Chondrocalcinosis of the AC joint was present in two patients. The subacromial space was decreased in all patients with complete obliteration of the space in three. The glenohumeral joint changes were classified subjectively as mild in three patients, moderate in five and severe in one (Table 1). Chondrocalcinosis of the glenohumeral joint was seen in one patient (Fig. 3B). On reviewing all available radiographs, one patient additionally had chondrocalcinosis of the knee (Patient no. 8).

Four cysts on conventional MRI had decreased signal intensity on $\mathrm{T} 1$ weighted images and increased signal intensity on T2-weighted images. In the two patients with direct MR arthrography, the signal intensity of the cyst on T1-weighted images was increased, consistent with the presence of gadolinium extending into the cyst. The inferior AC joint capsule was torn in all cases while the superior AC joint capsule was torn in only three, being extensively displaced with superior bulging by the cyst. In one patient there was free fluid around the cyst with the suspicion of cyst rupture (Fig. 1C). Extensive rotator cuff tears were present in four patients with at least two complete tendon tears and retraction (Table 1). In all six patients with MR imaging there was a clearly defined column of fluid extending from the glenohumeral joint into the AC joint and AC joint cyst (Fig. 2C). Varying degrees of fatty atrophy of the rotator cuff muscles were 

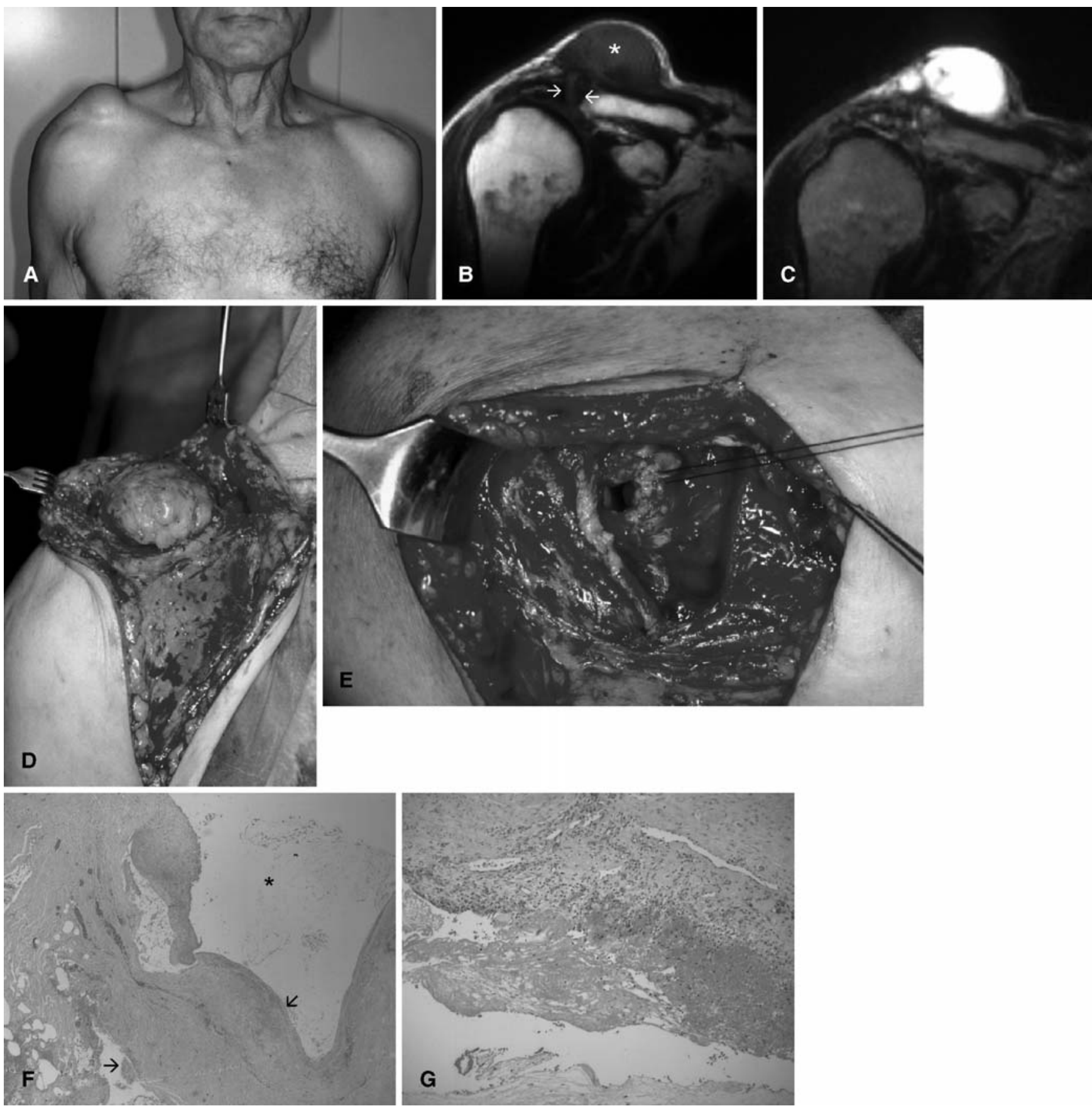

Fig. 1A-G 71-year-old male (Patient no. 5) presented with an enlarging painless mass of the shoulder region. A Characteristic clinical image of a right-sided AC joint mass. B MRI was performed for tumor staging. Oblique coronal T1 weighted image (TR 400, TE 20) shows the large AC joint associated supraclavicular cyst (*). Arrows mark the AC joint space. Complete supraspinatus and infraspinatus tendon tears are evident with articulation of the humeral head with the acromion. C Oblique coronal short tau inversion recovery (STIR) image (TR 3400, TE 15, TI 150m) shows

the typical homogeneous high signal within the cyst and some altered signal intensity in the adjacent soft tissue, suggesting a history of rupture. D Intraoperative image shows the superior surface of the AC joint cyst. $\mathbf{E}$ After resection of the cyst the communication with the AC joint is clearly seen. F Hemotoxylin and eosin stained histopathology slide at $2.5 \times$ magnification showing the cyst lumen (*), the synovial lining of the cyst and the thickened inflamed cyst wall (arrows). G At 10× magnification, the chronic inflammatory changes in the cyst wall and the fibrin are better visualized 

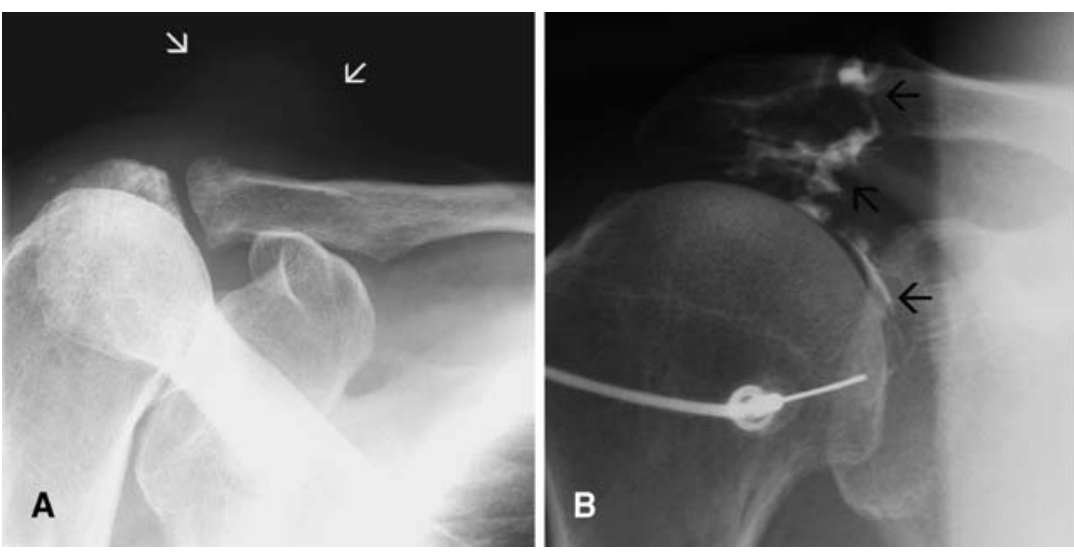

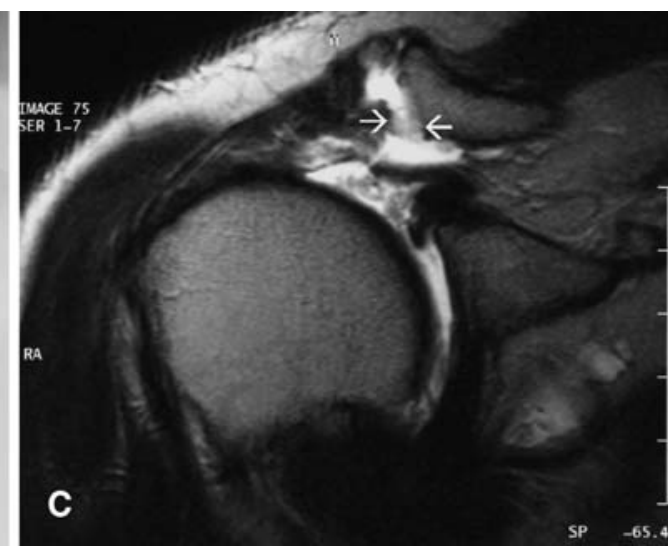

Fig. 2A-C 57-year-old male (Patient no. 1) presented with pain, shoulder mass and decreased movement of the shoulder joint. A Focused radiograph of the AC joint shows the soft tissue mass (arrows) and degenerative AC joint with small inferior clavicular osteophyte formation. B Arthrographic image showing the geyser sign (arrows). C Oblique coronal T2 weighted image (TR 3400, TE 85 ) shows the continuous column of joint fluid extending from the glenohumeral joint, through the extensive rotator cuff tear and the ruptured inferior $\mathrm{AC}$ joint capsule and ligament (arrows) into the AC joint

Table 1 Overview of patients with acromioclavicular (AC) joint cysts ( $S u b$ subscapularis, SS supraspinatus, IS infraspinatus, TM teres minor, $B i$ biceps brachii, $C P P D$ calcium pyrophosphate phosphate dihydrate)

\begin{tabular}{|c|c|c|c|c|c|c|c|c|c|}
\hline \multirow[t]{2}{*}{$\begin{array}{l}\text { Age/ } \\
\text { sex }\end{array}$} & \multicolumn{2}{|c|}{ Radiographs } & \multirow{2}{*}{$\begin{array}{l}\text { Arthrogram } \\
\overline{\text { Geyser sign }}\end{array}$} & \multicolumn{3}{|c|}{$\begin{array}{l}\text { MRI rotator cuff tendons and muscle } \\
\text { atrophy }\end{array}$} & \multirow[t]{2}{*}{ Management } & \multirow[t]{2}{*}{ Histology } & \multirow[t]{2}{*}{ Aspirate } \\
\hline & $\begin{array}{l}\text { Chondro- } \\
\text { calcinosis } \\
\text { of AC } \\
\text { joint }\end{array}$ & $\begin{array}{l}\text { Degenerative } \\
\text { changes } \\
\text { of gleno- } \\
\text { humeral joint }\end{array}$ & & $\begin{array}{l}\text { Partial- } \\
\text { thickness } \\
\text { tear }\end{array}$ & $\begin{array}{l}\text { Full-thick- } \\
\text { ness tear }\end{array}$ & $\begin{array}{l}\text { Fatty muscle } \\
\text { atrophy }\end{array}$ & & & \\
\hline $1.57 \mathrm{M}$ & & Moderate & + & & Sub, SS & Sub III, SS III & Surgery & \multirow{6}{*}{$\begin{array}{l}\text { Synovial } \\
\text { cyst }\end{array}$} & \\
\hline 2. $60 \mathrm{M}$ & & Moderate & & Sub, SS & & $\begin{array}{l}\text { Sub I, SS II, IS } \\
\text { IV }\end{array}$ & Surgery & & \\
\hline 4. $64 \mathrm{~F}$ & & Mild & + & Sub & SS, IS, & $\begin{array}{l}\text { Sub I, SS III, IS } \\
\text { III, }\end{array}$ & Surgery & & \\
\hline 5. $71 \mathrm{M}$ & & Moderate & & Sub & $\mathrm{SS}, \mathrm{IS}, \mathrm{Bi}$ & $\begin{array}{l}\text { Sub II, SS IV, IS } \\
\text { IV, }\end{array}$ & Surgery & & \\
\hline $6.68 \mathrm{M}$ & & Moderate & & Sub, IS & SS, TM, Bi & $\begin{array}{l}\text { Sub II, SS IV, IS } \\
\text { II, TM IV }\end{array}$ & Surgery & & \\
\hline 7. $68 \mathrm{M}$ & & Moderate & + & & & & $\begin{array}{l}\text { Aspirations, } \\
\text { sclerosing } \\
\text { synovectomy, } \\
\text { surgery }\end{array}$ & & $\begin{array}{l}\text { CPPD } \\
\text { crystals }\end{array}$ \\
\hline $9.86 \mathrm{M}$ & + & Mild & & & & & Conservative & $\begin{array}{l}\text { Synovial } \\
\text { cyst }\end{array}$ & \\
\hline
\end{tabular}

noted, with six patients having extensive rotator cuff tear associated with at least one muscle with a grade III or grade IV fatty atrophy (Table 1 ). One patient had a SLAP lesion. Two patients had a distended subacromial-subdeltoid bursa. There were no large glenohumeral joint effusions. The geyser sign [1] was positive in all the three patients who underwent arthrography (Fig. 2B). Ultrasound in one patient showed a cystic mass arising directly from the AC joint and the torn inferior AC joint capsule.
Operative and histopathological correlation

Seven patients successfully underwent surgery with correlation of imaging findings of the presence and degree of the rotator cuff tear, presence of the cyst and the joint communication. One patient referred from an outside institution had undergone repeat aspiration as well as radionuclide sclerosing synovectomy with persistent recurrence of the mass finally requiring surgical removal and shoulder prosthesis. Surgery included AC cyst and joint resection with subacromial and intra-articular debridement. The large rotator cuff tears were irreparable 
Fig. 3A-C 68-year-old male (Patient no. 8) who presented with a shoulder mass. A Clinical image of the shoulder mass. B Frontal radiograph with evidence of complete rotator cuff tear and marked degenerative changes in the glenohumeral joint with prominent inferior humeral head osteophyte formation and chondrocalcinosis (arrows). C Aspirate observed under polarised light microscopy shows weakly positive birefringent crystals. An inflamed synovial cyst was removed at surgery
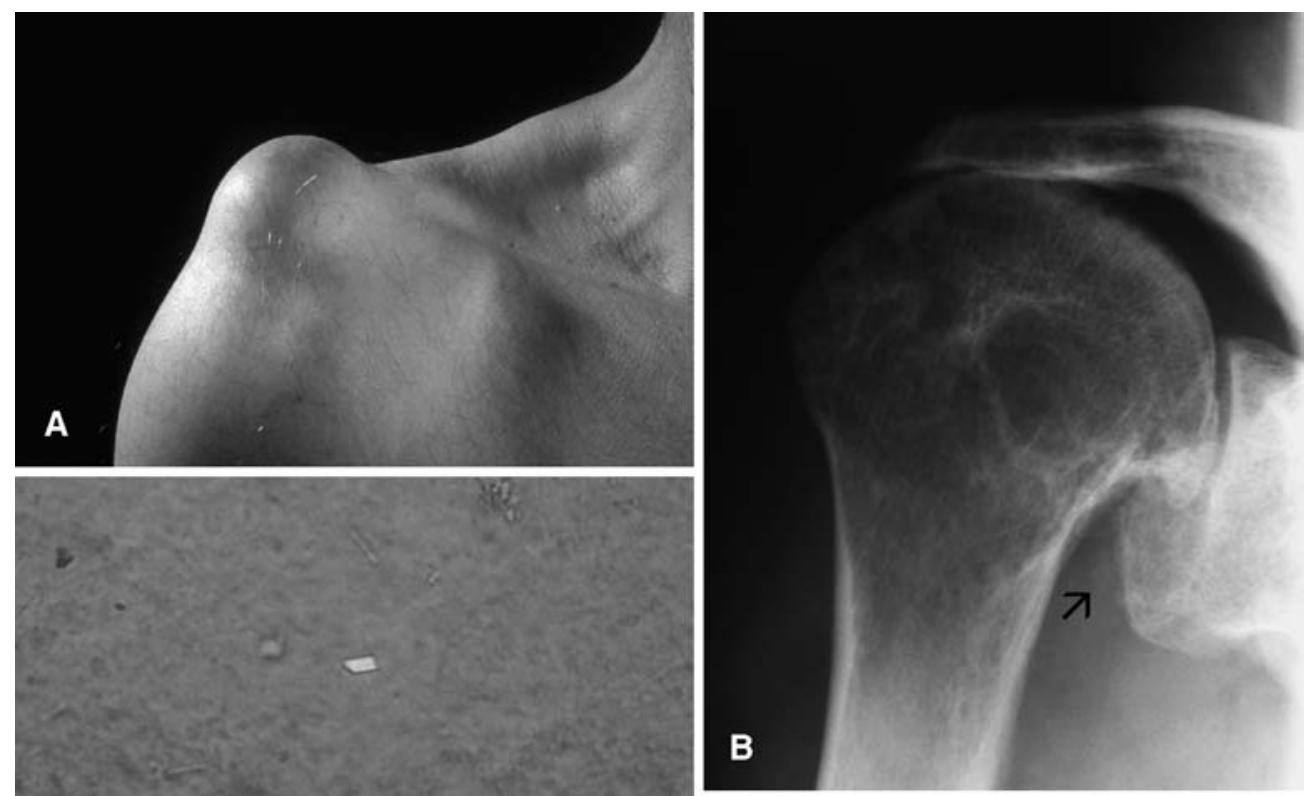

and required distal clavicular resection with resection interposition arthroplasty (deltoid and trapezius) or shoulder prosthesis. Histopathology revealed the synovial lining in two cysts with inflamed connective tissue surrounding the cyst lumen (Fig. 1F, G). Aspiration of the cyst was performed in two patients and CPPD crystals (Fig. 3C) were demonstrated in the aspirate. Two patients were managed conservatively.

\section{Discussion}

$\mathrm{AC}$ joint cysts are uncommon complications of degenerative rotator cuff tears. Patients are typically elderly and present with a swelling over the shoulder. The shoulder is often painless and the rotator cuff tear is functionally compensated. In this situation a malignant tumor has to be excluded [9]. With our series of nine cases we would like to stress that this pseudotumour of the shoulder is usually associated with extensive rotator cuff tears with a direct continuation of a column of fluid between the glenohumeral and $\mathrm{AC}$ joints extending into the AC cyst. Imaging, particularly MRI, excludes a more sinister tumor diagnosis. We also noted the presence of CPPD crystals within two of three AC joint cysts in patients with chondrocalcinosis, which has also rarely been reported in the rheumatological literature $[10,11,12]$.

The etiology of AC joint cyst is thought to be mechanical. Additional information has been gained from arthrography [1]. The geyser sign on shoulder arthrography is characterized by leakage of contrast from the gle- nohumeral joint into the subacromial-subdeltoid bursa and from there into the AC joint space. It usually indicates a full thickness tear of the rotator cuff of long duration. The pathomechanics of the geyser sign have been described [1]. The muscle belly of the supraspinatis tendon lies directly under the $\mathrm{AC}$ joint with only a thin layer of fat and bursa separating the joint space from the rotator cuff. During arm elevation, the rotator cuff tendons pass directly under the anterior acromion, coracoacromial arch and the $\mathrm{AC}$ joint. For the geyser sign to occur, there must be erosion of the inferior AC joint capsule. This erosion may occur within the capsule from a gradually enlarging osteophyte from a post-traumatic or degenerative AC joint or from undersurface mechanical erosion as the humeral head rubs against the AC joint or from a combination of both. Attempts to elevate the arm will push the humeral head against the anterior acromion and AC joint increasing the impingement. With increasing tear the external rotators are weakened more than the internal rotators and the greater tuberosity moves more medially and superiorly, with further traumatisation of the undersurface of the AC joint. The impingement is believed to cause gradual erosion of the inferior AC joint capsule. When contrast is injected during arthrography, it rises through the defect in the rotator cuff and the inferior AC joint capsule to produce the geyser sign on the arthrogram.

The first cases of $\mathrm{AC}$ joint cysts associated with rotator cuff tears were presented by Craig in 1986 [2]. He described two patients with AC joint cysts. One of them had been repeatedly treated by aspiration of the cyst and steroid injections but finally required surgical excision 
due to cyst recurrence. Radiographs showed the degeneration in the AC joint and glenohumeral changes compatible with a rotator cuff tear. Arthrography demonstrated the geyser sign. The other patient had been treated for a massive cuff tear and degenerative changes in the shoulder with shoulder arthrodesis. Double contrast arthrography demonstrated the communication between the glenohumeral and $\mathrm{AC}$ joint. The attempt to treat the cyst alone without evaluation of the rotator cuff status will almost always result in recurrence of the cyst, as has been confirmed by other authors in the orthopaedic literature $[3,4,13]$. This was also seen in one patient in our series.

Another series of three patients with AC joint cysts in association with rotator cuff tears was reported by Postachinni et al. in 1993 [4]. The authors stated that the condition was rare and stressed again the necessity for evaluation of the rotator cuff status. In addition they advocated resection of the lateral end of the clavicle when the cuff tear cannot be repaired.

Cvitanic et al. in 1999 [9] described the MR arthrographic appearance of an AC joint cyst with demonstration of the glenohumeral communication. They stated that conventional arthrography was inconsistent in revealing the communication between the glenohumeral joint and the AC joint. MR arthrography was performed immediately following intra-articular contrast material administration, which revealed the communication after a false negative single contrast arthrogram. A false negative conventional arthrogram could result from compression of the communication with enlargement of the cyst, from inspissation of the fluid and blockage of the communication or due to the viscid nature of the fluid, which prevents mixing and spreading of the contrast material.
They concluded that MRI without enhancement was adequate to make the diagnosis of $\mathrm{AC}$ joint cysts associated with rotator cuff tear, even though ineffective in revealing communications between cysts and joints. We have been able to visualise the joint capsule and fluid continuation in all the patients who underwent MRI and therefore feel confident with a non-arthrographic MRI diagnosis.

CPPD deposition disease is a relatively common arthropathy that occurs mostly in elderly people [10]. The knee, wrist, hip and shoulder are frequently involved, in that order. It is not a surprise that three of our patients had CPPD deposition disease, as this arthropathy is frequently associated with rotator cuff tear [14].

In conclusion, a soft tissue mass arising over the acromioclavicular joint may be the first presentation of a chronic extensive rotator cuff tear, representing communication of fluid between the glenohumeral joint and acromioclavicular joints. MRI can demonstrate the associated rotator cuff tear and cyst. Since CPPD crystal deposition disease is also associated with rotator cuff tears and seen in older individuals, it may be seen in shoulders with these cysts as demonstrated in three of our patients. Potential limitations and sources of error related to this study are the small number of patients and the retrospective study design. The unusual feature of this study is that all patients presented with a mass for tumor imaging. This shows that this entity should be kept in mind when a patient comes for evaluation of a swelling over the shoulder joint, as the clinical features of a rotator cuff tear may not always be evident.

Acknowledgement We thank Dr. Thomas Lehmann for his contribution and Susanne Furrer for manuscript support.

\section{References}

1. Craig EV. The Geyser sign and torn rotator cuff: clinical significance and pathomechanics. Clin Orthop 1984; 191:213-15.

2. Craig EV. The acromioclavicular joint cyst. An unusual presentation of rotator cuff tear. Clin Orthop 1986; Jan (202):189-92.

3. Marino AJ, Tyrrell PN, El-Houdiri Y, Kelly CP. Acromioclavicular joint cyst and rotator cuff tear. J Shoulder Elbow Surg 1998; 7:435-37.

4. Postacchini F, Perugia D, Gumina S. Acromioclavicular joint cyst associated with rotator cuff tear. A report of three cases. Clin Orthop 1993; 294:111-13.

5. Bigliani LU, Morrison DS, April EW. The morphology of the acromion and its relationship to rotator cuff tears. Orthop Trans 1986; 10:228.
6. Brossmann J, Czerny C, Freyschmidt J. Akromioklivikulargelenk. In: Freyschmidt's "Köhler/Zimmer" Grenzen des Normalen und Anfänge des Pathologischen in der Radiologie des kindlichen und erwachsenen Skeletts. Stuttgart: Thieme Verlag; 2001:285-89.

7. Kotzen LM. Roentgen diagnosis of rotator cuff tear: report of 48 surgically proven cases. AJR 1971; 112:507.

8. Goutalier D, Postel J-M, Bernageau J, Lavau L, Voisin M-C. Fatty muscle degeneration in cuff ruptures. Clin Orthop 1994; 304:78-83.

9. Cvitanic O, Schimandle J, Cruse A, Minter J. The acromioclavicular joint cyst: Glenohumeral communication revealed by MR Arthrography. J Comput Assist Tomogr 1999; 23:141-43.

10. Huang GS, Bachmann D, Taylor JAM, Marcelis S, Haghighi P, Resnik D. Calcium pyrophosphate dihydrate crystal deposition disease and pseudogout of the acromioclavicular joint: radiographic and pathologic features. J Rheumatol 1993; 12:2077-82.
11. Gerster JC. Cystic distension of the acromioclavicular joint in calcium pyrophosphate dihydrate crystal deposition disease. J Rheumatol 1995; 22:371-72.

12. Cooper AM, Hayward C, Williams BD. Calcium pyrophosphate deposition disease-involvement of the acromioclavicular joint with pseudocyst formation. Br J Rheumatol 1993; 32:248-50.

13. Groh GI, Badwey TM, Rockwood CR. Treatment of cysts of the acromioclavicular joint with shoulder hemiarthroplasty. The Journal of Bone Joint Surg Am 1993; 75:1790-94.

14. Steinbach LS. CPPD crystal deposition disease: imaging characteristics. RCNA 2004; 42:185-206. 